\title{
Remifentanil Has Sufficient Hypnotic and Amnesic Effect for Induction of Anesthesia by Itself
}

\author{
Takeshi Yokoyama $^{1}$, Eiji Sakamoto ${ }^{1}$, Fumiyasu Yamasaki ${ }^{2}$, Koichi Yamashita $^{3}$, Tomoaki Yatabe ${ }^{3}$, \\ Kunio Suwa ${ }^{4}$
}

${ }^{1}$ Department of Dental Anesthesiology, Faculty of Dental Science, Kyushu University, Fukuoka, Japan; ${ }^{2}$ Department of Clinical Laboratory, Kochi Medical School Hospital, Nankoku, Japan; ${ }^{3}$ Department of Anesthesiology and Critical Care Medicine, Kochi Medical School Hospital, Nankoku, Japan; ${ }^{4}$ Teikyo Junior College, Tokyo, Japan.

Email: yokoyama@dent.kyushu-u.ac.jp

Received November $6^{\text {th }}, 2013$; revised December $12^{\text {th }}, 2013$; accepted January $11^{\text {th }}, 2014$

Copyright (C) 2014 Takeshi Yokoyama et al. This is an open access article distributed under the Creative Commons Attribution License, which permits unrestricted use, distribution, and reproduction in any medium, provided the original work is properly cited. In accordance of the Creative Commons Attribution License all Copyrights @ 2014 are reserved for SCIRP and the owner of the intellectual property Takeshi Yokoyama et al. All Copyright (C) 2014 are guarded by law and by SCIRP as a guardian.

\section{ABSTRACT}

With a small-dose remifentanil, some patients showed no reaction and did not remember it postoperatively. We, therefore, hypothesized that remifentanil may decrease the level of consciousness and/or exhibit amnesic effect when stimulations are avoided. Thirty-patients were divided into two groups: non-stimulation group and stimulation group. Anesthesia was induced with $1 \mathrm{micro}-\mathrm{g} \mathrm{kg}^{-1} \cdot \mathrm{min}^{-1}$ of remifentanil using no additional hypnotic agent. In the non-stimulation group, patients were left free from any stimulation except non-invasive blood pressure monitoring. In the stimulation group, patients were asked to follow verbal commands. The level of consciousness was evaluated with electroencephalogram and BIS-value derived from it. In the non-stimulation group, all patients reached the decreased level of consciousness in 5 minutes. In the stimulation group, however, 14 patients were judged to be still conscious. 10 patients could open their mouth at the 5th minute, but 9 of these 10 patients did not remember it postoperatively. In conclusion, remifentanil, with no additional anesthetics, exhibited hypnotic and amnesic effects when stimulations were kept minimal.

\section{KEYWORDS}

Remifentanil; Hypnotic potential; Stimulation; Amnesia

\section{Introduction}

We experienced several cases of severe muscle rigidity during induction of anesthesia using remifentanil. It has been reported that fentanyl-induced rigidity was accompanied by unconsciousness [1]. Therefore, remifentanilinduced rigidity may also be related to decrease in the level of consciousness. In several other cases, we observed decrease in Bispectral Index (BIS) value with clear delta waves in the electroencephalogram (EEG) only by 1 micro-g $\cdot \mathrm{kg}^{-1} \cdot \mathrm{min}^{-1}$ of infusion of remifentanil in a few minutes since the start of infusion, and those patients commented after surgery that they were unconscious and did not remember the situation during induction of anesthesia. We, therefore, hypothesized that remifentanil may have hypnotic and amnesic effect and may decrease the level of consciousness, and verbal stimulation may attenuate this hypnotic effect. Assuming that BIS value and EEG pattern present the level of consciousness, we investigated the effect of verbal stimulation on the BIS value during induction of anesthesia in order to characterize the hypnotic and amnesic effect of remifentanil.

\section{Methods}

\subsection{Patients}

The institutional Review Board of Kochi Medical School Hospital approved the study, and written informed consent was obtained from patients before they were enrolled. We recruited patients undergoing elective surgery under general anesthesia. They were aged between 
20 and 80 years and ASA physical status I or II. Those patients were divided into two groups, with or without verbal commands. Patients were excluded if they had a history of epileptic disease and/or were taking cerebrally active medications, or if they were agitated with anxiety.

\subsection{Anesthesia}

Patients did not receive any premedication since the previous day, and they slept well at night. Electrocardiogram (ECG), pulse oxymetry $\left(\mathrm{SpO}_{2}\right)$ and Bispectral Index (BIS) using a BIS monitor (A2000Xp, Aspect Medical Systems, USA) were monitored. They were asked to open their mouth once before the start of induction of anesthesia. After 2 minutes of oxygenation with $6 \mathrm{~L} / \mathrm{min}$ of $100 \%$ oxygen, 5 micro-g $\mathrm{kg}^{-1}$ of atropine were given intravenously and continuous infusion of remifentanil at 1 micro-g $\mathrm{kg}^{-1} \cdot \mathrm{min}^{-1}$ was started. The operating room was kept quiet. During induction, patients of the nonstimulation group (NS group) were kept free from any stimulation except non-invasive blood pressure monitoring at every minutes, while those of the stimulation group (S group) were asked to grip hands, to open eyes and to take deep-breaths every fifteen seconds. They were also asked to open mouth twice at the 3rd minute and the 5th minute after the start of remifentanil infusion. When BIS value decreased to less than 60 with clear monorhythmic delta waves in the EEG, we judged the level of consciousness decreased sufficiently, and this timing was noted. Then, $0.1 \mathrm{mg} \cdot \mathrm{kg}^{-1}$ of vecuronium was give intravenously. The trachea was intubated $3-4$ minutes after vecuronium was administered. Anesthesia was maintained with only remifentanil for 2 minutes, and sevoflurane in oxygen was given thereafter. If BIS value did not decrease less than 60 in 5 minutes from the start of remifentanil infusion, sevoflurane (3\% - 5\% in $6 \mathrm{~L}$ of oxygen) was started. With the decrease in BIS value, 0.1 $\mathrm{mg} \cdot \mathrm{kg}^{-1}$ of vecuronium was given intravenously, and tracheal intubation was performed 3 - 4 minutes thereafter.

Postoperatively all patients were interviewed twice, in the recovery room when the patient regained consciousness and at the post-round on the next day. The patients were asked if they had any memory during induction of anesthesia, especially what they remembered before they lost consciousness. In addition, patients in the $\mathrm{S}$ group were asked whether they had memory of mouth opening at the 3rd minute and at the 5th minute. The patients were asked again if they remembered anything during induction of anesthesia by telephone interview after their discharge. The serum concentration (SC) and the effectsite concentration (ESC) were estimated using TIVA Trainer $^{\mathrm{TM}}$ ver.8. In the case that BIS value decreased to less than 60 with clear monorhythmic delta waves in 5 minutes, the median effective dose $\left(\mathrm{ED}_{50}\right)$ of remifentanil for decreased level of consciousness was calculated from the time of remifentanil infusion, and the median effective SC $\left(\mathrm{EC}_{50}\right)$ was also estimated.

\subsection{Statistical Analysis}

Chai square test was used to evaluate the difference for its level of significance of hypnotic effect between groups, namely whether the decrease in the level of consciousness with BIS value less than 60 and clear monorhythmic delta waves was obtained in 5 minutes or not. ANOVA repeated measures were used to analyze the difference of blood pressure and heart rate between groups, and post-hoc Sheffe test was used to analyze the change in blood pressure and heart rate during induction of anesthesia in each group. The Student's paired t test was used to analyze the patient's background and estimated remifentanil concentrations. Mann-Whitney U-test with Bonferroni correction was used to analyze the duration for appearance of delta waves from the start of the remifentanil infusion between groups. A $p$ value $<0.05$ was considered as the level of statistical significance.

\section{Results}

Thirty patients completed this study. There were no significant differences in the demographic background (age, gender, height and weight) of patients between the NS group $(n=15)$ and the $S$ group $(n=15)$ (Table 1$)$.

\subsection{Hypnotic Effect of Remifentanil}

In the NS group, all patients reached the BIS value of 60 or below with clear monorhythmic delta waves in the EEG in 5 minutes (Table 2). Delta waves appeared within 4 minutes in all patients (Figure 1). The BIS decreased to less than 60 in 5 minutes from the start of remifentanil administration. The mean SC and the mean ESC were estimated to be 16.4 (SD: 2.7$) \mathrm{ng} \cdot \mathrm{ml}^{-1}$ and 11.1 (SD: 1.4$) \mathrm{ng} \cdot \mathrm{ml}^{-1}$, respectively. $\mathrm{ED}_{50}$ was at $3.8 \mathrm{mg} \cdot \mathrm{kg}^{-1}$, and the serum $\mathrm{EC}_{50}$ was estimated to be $17.1 \mathrm{ng} \cdot \mathrm{ml}^{-1}$, and the $\mathrm{EC}_{50}$ in the effective tissue was estimated to be $11.7 \mathrm{ng} \cdot \mathrm{ml}^{-1}$. Tracheal intubation was performed in 549 (SD: 54) seconds after the start of remifentanil administration. The SC and the ESC were estimated to be

Table 1. Characteristics of patients of the NS group and the $\mathrm{S}$ group. Values are mean (SD [range]) or mean (SD).

\begin{tabular}{ccc}
\hline & NS group $(\mathrm{n}=15)$ & S group $(\mathrm{n}=15)$ \\
\hline Age; years & $51(18[22-78])$ & $53(13[32-70])$ \\
Weight; $\mathrm{kg}$ & $57(10)$ & $60(9)$ \\
Height; $\mathrm{cm}$ & $160(7)$ & $162(6)$ \\
Sex; M:F & $7: 8$ & $8: 7$ \\
\hline
\end{tabular}


Table 2. Effect of remifentanil on BIS and EEG. Values are mean (SD) or number (proportion).

\begin{tabular}{|c|c|c|c|c|}
\hline & $\begin{array}{l}\text { Appearance delta } \\
\text { waves }\end{array}$ & $\begin{array}{c}\text { Duration delta } \\
\text { waves (s) }\end{array}$ & BIS $<60$ & $\begin{array}{l}\text { Duration BIS } \\
<60 \text { (s) }\end{array}$ \\
\hline NS group & 15 (100\%) & $182(30)$ & 15 (100\%) & 237 (33) \\
\hline S group & $8(53 \%)^{*}$ & $240(32)^{*}$ & $1(6.7 \%)^{*}$ & 225 \\
\hline
\end{tabular}

Appearance delta waves: patients with delta waves in the EEG in 5 minutes. Duration delta waves (s): duration until appearance of delta waves from the start of remifentanil infusion. BIS < 60: patients with BIS value decreased less than 60 in 5 minutes. Duration BIS $<60$ (s): duration until BIS value decreased less than $60 .{ }^{*} p<0.05$ vs NS group.

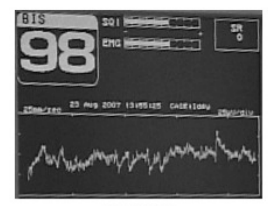

baseline

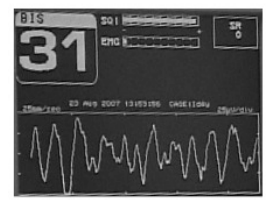

Pre-TI

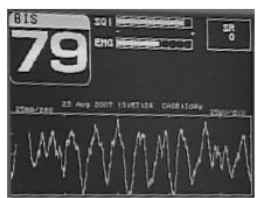

$120 \mathrm{sec}$.

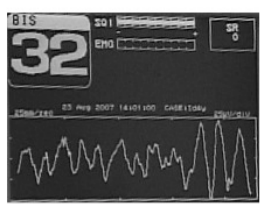

Post-TI

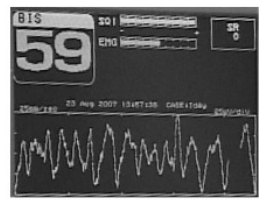

BIS $<60$ (185 sec.)
Figure 1. A typical changes in the EEG (53-year-old woman).

21.4 (SD: 3.5) ng. $\mathrm{ml}^{-1}$ and 19.5 (SD: 3.0) $\mathrm{ng} \cdot \mathrm{ml}^{-1}$, respectively. EEG pattern did not changed between before and after tracheal intubation. In the S group, on the other hand, only one patient reached the BIS value of 60 or below with clear monorhythmic delta waves in the EEG in 5 minutes. In 8 of 15 patients, delta waves in the EEG were observed in 5 minutes. The delta waves in this group were unstable, and in 3 out of these 8 patients they disappeared at the 5 th minute. The time required for the appearance of delta waves in these 8 patients was significantly longer than that of the NS group ( $p=0.0002)$. BIS values of other 14 patients remained higher than 60; 87 (SD: 7) at the 5th minute. All patients could open their mouth at the 3rd minute, but mouth openings of 14 patients were clearly smaller and the reactions were slower than before initiation of remifentanil infusion. One patient could open his mouth as wide as and as quick as he did before remifentanil infudsion. 10 of 15 patients could open their mouth even at the 5th minute, but the mouth openings were always smaller and the reactions were slower. 4 patients responded with something to verbal command, but could not open their mouth. The patient, whose BIS value decreased to less than 60 , showed no response to verbal commands. The estimated SC of remifentanil at the 5th minute or reaching the BIS value of 60 or below with clear monorhythmic delta waves was 17.5 (SD: 3.1 ) ng. $\mathrm{ml}^{-1}$ and the ESC was 13.5 (SD: 2.0) $\mathrm{ng} \cdot \mathrm{ml}^{-1}$. The estimated SC of remifentanil at tracheal intubation was 21.1 (SD: 3.6) $\mathrm{ng} \cdot \mathrm{ml}^{-1}$ and the ESC was 19.6 (SD: 3.3$) \mathrm{ng} \cdot \mathrm{ml}^{-1}$. There were no significant differences in the serum and ESC of remifentanil when we judged the level of consciousness decreased sufficiently or at the 5th minute and at tracheal intubation between groups.

\subsection{Amnesic Effect of Remifentanil}

In the NS group, all patients commented that they remembered coming to the operating room and having a mask placed on their faces, and felt sleepy quickly after the start of remifentanil administration, and they had no memory of passive ventilation, laryngoscopy or tracheal intubation.

In the S group, all 15 patients could open their mouths at the 3rd minute from the start of remifentanil infusion, and 10 patients could open their mouths even at the 5th minute. The majority of the patients, however, did not remember it (Table 3). In the telephone interview after their discharge, they could not remember anything additional.

\subsection{Blood Pressure and Heart Rate}

There was a significant difference in systolic blood pressure during induction of anesthesia between groups ( $p=$ 0.0461), while the difference in diastolic blood pressure and heart rate did not reach to the significant level. In the NS group, there were no significant differences in the changes of systolic and diastolic blood pressure during induction of anesthesia; before induction, when BIS value decreased to less than 60, and before and after tracheal intubation (Table 4). The heart rate increased significantly after tracheal intubation. In the S group, the blood pressure decreased after inhalation of sevoflurane, but the heart rate did not change significantly during induction of anesthesia.

\section{Discussion}

In the case of natural sleep at night, verbal stimulation often disturbs one's falling asleep. Even auditory evoked

Table 3. Memory of mouth opening during induction (S group).

\begin{tabular}{ccc}
\hline & $\begin{array}{c}\text { First mouth } \\
\text { opening (3min) }\end{array}$ & $\begin{array}{c}\text { Second mouth } \\
\text { opening (5min) }\end{array}$ \\
\hline Memory $(+)$ & $5(/ 15)$ & $1(/ 10)$ \\
Memory $(-)$ & $10(/ 15)$ & $9(/ 10)$ \\
\hline
\end{tabular}

Memory $(+)$ : Number of patients remembered mouth opening (/number of patients performed mouth opening) at emergence of anesthesia after surgery, at the post-round on the next day or telephone interview after discharge (3 6 months). Memory(-): Number of patients without memory of mouth opening (/number of patients performed mouth opening). All 15 patients could open their mouth at 3rd min and 10 patients still could open at 5th min from the start of remifentanil infusion. 
Table 4. Alteration of blood pressure and heart rate. Values are mean (SD).

\begin{tabular}{cccccc}
\hline & & Baseline & $\begin{array}{c}\text { BIS }<60 \\
\text { or 5min }\end{array}$ & pre-TI & post-TI \\
\hline \multirow{2}{*}{ sBP } & NS group & $130(14)$ & $129(12)$ & $131(19)$ & $136(14)$ \\
& S group & $134(14)$ & $130(20)$ & $103(15)^{* \#}$ & $109(13)^{* \#}$ \\
& NS group & $71(9)$ & $70(8)$ & $69(13)$ & $75(12)$ \\
dBP & S group & $78(9)$ & $73(11)$ & $56(12)^{* \#}$ & $58(12)^{* \#}$ \\
& NS group & $73(12)$ & $75(18)$ & $77(15)$ & $93(17)^{* \# \mathbf{S}}$ \\
\multirow{2}{*}{ HR } & S group & $70(11)$ & $74(19)$ & $69(14)$ & $76(15)$ \\
\hline
\end{tabular}

Baseline: before induction, BIS $<60$ or $5 \mathrm{~min}$ : at loss of consciousness, or at the 5th minute from the start of remifentanil infusion when the patient did not archive BIS $<60$ in 5 minutes, pre-TI: before tracheal intubation, postTI: after tracheal intubation. ${ }^{*} p<0.05$ vs cont; ${ }^{\#} p<0.05$ vs LOS or $5 \mathrm{~min} ;{ }^{\mathrm{s}} p$ $<0.05$ vs pre-TI.

potential click sound increases BIS value and entropy during propofol sedation [2]. When we kept stimulation to minimal, we experienced several patients with decreased BIS value of less than 60 during induction of anesthesia only with less than 5 micro-g. $\mathrm{kg}^{-1}$ of remifentanil before the current study. When we called to those patients, their responses were small or none. We, therefore, hypothesized that verbal stimulation attenuates the hypnotic effect during induction of anesthesia using remifentanil. The current study examines the action of remifentanil on hypnosis and amnesia, and investigates further the effect of verbal stimulation on hypnosis and amnesia. We observed that remifentanil induced monorhythmic delta waves in the EEG and decreased BIS value when we kept stimulation to minimal. Tracheal intubation was performed uneventfully with remifentanil and vecuronium. However, stimulation with verbal commands disturbed or delayed the appearance of monorhythmic delta waves and a decrease in BIS value. Thus, the hypnotic effect may be attenuated easily by verbal commands. In addition, our data revealed that remifentanil exhibits amnesic effect by itself.

We excluded patients with epilepsy, since there are reports of seizures related with this agent $[3,4]$. Patients complaining of anxiety were also excluded, since they could not have slept well on the previous night, and their blood pressure and heart rate before induction of anesthesia usually increased by more than $20 \%$ of usual values. Thus, the candidates for this study were not many and the range of the age was wide, but there was no difference in the time for appearance of delta waves and BIS decreased to less than 60 between elderly and young patients although remifentanil is more potent in elderly patients than in young patients. In the preliminary trials of this study, some patients with severe anxiety of operation showed increase in blood pressure and seizure-like reaction after the start of remifentanil infusion. In the current study, 5 patients showed sign of seizure-like reaction and/or rigidity after the start of remifentanil infusion. Thus, they were received $0.1 \mathrm{mg} \cdot \mathrm{kg}^{-1}$ of midazolam and were excluded from this study. Jhaveri's reported that the $\mathrm{ED}_{50}$ of remifentanil for LOC was $12 \mathrm{mi}-$ cro-g. $\mathrm{kg}^{-1}$, and remifentanil less than 5 micro-g $\mathrm{kg}^{-1}$ did not produce LOC [5]. In their study, however, the hypnotic effect was examined by failure to respond to verbal stimulation asking to open eyes and take a deep breath every 10 seconds. In our study, BIS monitor was useful for avoiding stimulation, and may add information for judging a decrease in consciousness [6-8], although BIS may be less reliable for sedative effects by opioids [9-12].

In the NS group, monorhythmic delta waves appeared earlier than that of the $\mathrm{S}$ group and BIS values decreased to less than 60 in 3 - 5 minutes. In the $S$ group, however, delta waves in the EEG were observed in 8 of 15 patients in 5 minutes, and these delta waves were unstable. Fourteen of 15 patients did not reach the BIS value of 60 or below with clear monorhythmic delta waves in the EEG, although electromyographic signal affected the BIS value [13]. In the NS group, all patients were intubated without causing changes in the EEG and blood pressure, and total infusion dose of remifentanil was 9.2 (SD: 0.9) micro$\mathrm{g} \cdot \mathrm{kg}^{-1}$ at intubation. These results may indicate that remifentanil exhibited hypnotic effect, and suggest that the hypnotic effect is exhibited effectively when stimulation was kept minimal. The $\mathrm{ED}_{50}$ for decreased BIS value less than 60 was 3.8 micro-g. $\mathrm{kg}^{-1}$ and $\mathrm{EC}_{50}$ were estimated to be $17.1 \mathrm{ng} \cdot \mathrm{ml}^{-1}$. In some cases, however, BIS value decreased less than 60 once, then it increased momentarily by a weak stimulus such as mask ventilation. The sedative state induced by remifentanil is so sensitive to stimulation that it may be interrupted quite easily. We may speculate that such characteristics may often lead to awareness during anesthesia [14].

Amnesic potential of remifentanil has not been reported yet, while morphine-induced amnesia in animal model has been known well $[15,16]$. In the $S$ group, all 15 patients opened their mouths at the 3rd minute, but 10 of them did not remember their mouth opening. Even at the 5th minute, 10 patients could open their mouth, but 9 patients did not remember it. We interpret these findings as indicating that remifentanil has amnesic effect. All patients of the NS group commented that they lost consciousness just after feeling warm, and felt it a short period within one or two minutes. These results also indicate that remifentanil might have amnesic effect and/or might have achieved LOC.

The effects of opioids on cardiovascular variables are usually less when it was used without other anesthetics [17], while propofol and inhalation anesthetics suppress cardiac function and dilate vessels, and often cause 
hypotension [18-20]. In the NS group, the blood pressure did not change significantly during induction, while the heart rate after the intubation increased by $27 \%$ compared to that before the remifentanil infusion. Although the heart rate did not change significantly in the S group, systolic blood pressure decreased significantly after inhalation of sevoflurane. These results showed that remifentanil does not cause hypotension by itself, and bradycardia can be prevented by 5 micro-g $\mathrm{kg}^{-1}$ of atropine.

\section{Conclusion}

In conclusion, remifentanil decreases the level of consciousness and causes amnesia by itself. By avoiding stimulation effectively, we successfully induced anesthesia and intubated the trachea smoothly without any supplementary hypnotics, and patients had no memory of being intubated.

\section{REFERENCES}

[1] J. B. Streisand, P. L. Bailey, L. LeMaire, M. A. Ashburn, S. D. Tarver, J. Varvel and T. H. Stanley, "FentanylInduced Rigidity and Unconsciousness in Human Volunteers. Incidence, Duration, and Plasma Concentrations,” Anesthesiology, Vol. 78, No. 4, 1993, pp. 629-634. http://dx.doi.org/10.1097/00000542-199304000-00003

[2] T. Nishiyama, "The Effects of Auditory Evoked Potential Click Sounds on Bispectral Index and Entropy,” Anesthesia \& Analgesia, Vol. 107, No. 2, 2008, pp. 545-548. http://dx.doi.org/10.1213/ane.0b013e318174deed

[3] G. W. Haber and R. S. Litman, "Generalized Tonic-Clonic Activity after Remifentanil Administration," Anesthesia \& Analgesia, Vol. 93, No. 6, 2001, pp. 1532-1533. http://dx.doi.org/10.1097/00000539-200112000-00040

[4] J. Nielsen and M. Krøigaard, "Seizures in a 77-Year-Old Woman after a Bolus Dose of Remifentanil," Acta Anaesthesiologica Scandinavica, Vol. 48, No. 2, 2004, pp. 253-254.

http://dx.doi.org/10.1111/j.0001-5172.2004.00294.x

[5] R. Jhaveri, P. Joshi, R. Batenhorst, V. Baughman and P. S. Glass, "Dose Comparison of Remifentanil and Alfentanil for Loss of Consciousness,” Anesthesiology, Vol. 87, No. 2, 1997, pp. 253-259. http://dx.doi.org/10.1097/00000542-199708000-00011

[6] J. C. Sigl and N. G. Chamoun, "An Introduction to Bispectral Analysis for the Electroencephalogram,” Journal of Clinical Monitoring and Computing, Vol. 10, No. 6, 1994, pp. 392-404.

[7] P.S. Glass, M. Bloom, L. Kearse, C. Rosow, P. Sebel and P. Manberg, "Bispectral Analysis Measures Sedation and Memory Effects of Propofol, Midazolam, Isoflurane, and Alfentanil in Healthy Volunteers," Anesthesiology, Vol. 86, No. 4, 1997, pp. 836-847. http://dx.doi.org/10.1097/00000542-199704000-00014

[8] M. J. Griffiths, A. W. Preece and J. L. Green, "Monitoring Sedation Levels by EEG Spectral Analysis," Anesthe- sia Progress, Vol. 38, No. 6, 1991, pp. 227-231.

[9] G. Barr, R. E. Anderson, A. Owall and J. G. Jakobsson, "Effects on the Bispectral Index during Medium-High Dose Fentanyl Induction with or without Propofol Supplement,” Acta Anaesthesiologica Scandinavica, Vol. 44, No. 7, 2000, pp. 807-811.

http://dx.doi.org/10.1034/j.1399-6576.2000.440707.x

[10] S. C. Manyam, D. K. Gupta, K. B. Johnson, J. L. White, N. L. Pace, D. R. Westenskow and T. D. Egan, "When Is a Bispectral Index of 60 too Low?: Rational Processed Electroencephalographic Targets Are Dependent on the Sedative-Opioid Ratio,” Anesthesiology, Vol. 106, No. 3, 2007, pp. 472-483.

http://dx.doi.org/10.1097/00000542-200703000-00011

[11] D. K. Gupta, "Processed EEG (Bispectral Index) Is Not Appropriate for Characterizing Opioid Sedation," Clinical Pharmacology \& Therapeutics, Vol. 83, No. 5, 2008, pp. 667-668. http://dx.doi.org/10.1038/sj.clpt.6100265

[12] A. Yli-Hankala, "Awareness Despite Low Spectral Entropy Values,” Anesthesia \& Analgesia, Vol. 106, No. 5, 2008, p. 1585. http://dx.doi.org/10.1213/ane.0b013e31816a1a86

[13] J. Bruhn, T. W. Bouillon and S. L. Shafer, "Electromyographic Activity Falsely Elevates the Bispectral Index,” Anesthesiology, Vol. 92, No. 5, 2000, pp. 1485-1487. http://dx.doi.org/10.1097/00000542-200005000-00042

[14] K. B. Domino, K. L. Posner, R. A. Caplan and F. W. Cheney, "Awareness during Anesthesia: A Closed Claims Analysis,” Anesthesiology, Vol. 90, No. 4, 1999, pp. 1053-1061.

http://dx.doi.org/10.1097/00000542-199904000-00019

[15] M. Nishimura, Y. Shiigi and H. Kaneto, "State Dependent and/or Direct Memory Retrieval by Morphine in Mice," Psychopharmacology (Berl), Vol. 100, No. 1, 1990, pp. 27-30. http://dx.doi.org/10.1007/BF02245784

[16] M. R. Zarrindast and A. Rezayof, "Morphine StateDependent Learning: Sensitization and Interactions with Dopamine Receptors," European Journal of Pharmacology, Vol. 497, No. 2, 2004, pp. 197-204. http://dx.doi.org/10.1016/j.ejphar.2004.06.041

[17] J. Nauta, S. de Lange, D. Koopman, J. Spierdijk, J. van Kleef and T. H. Stanley, "Anesthetic Induction with Alfentanil: A New Short-Acting Narcotic Analgesic,” Anesthesia \& Analgesia, Vol. 61, No. 3, 1982, pp. 267-272.

[18] R. J. Fragen, E. H. Hanssen, P. A. Denissen, L. H. Booij and J. F. Crul, "Disoprofol (ICI 35868) for Total Intravenous Anaesthesia,” Acta Anaesthesiologica Scandinavica, Vol. 27, No. 2, 1983, pp. 113-116. http://dx.doi.org/10.1111/j.1399-6576.1983.tb01918.x

[19] J. C. Raeder and G. Misvaer, “Comparison of Propofol Induction with Thiopentone or Methohexitone in Short Outpatient General Anaesthesia,” Acta Anaesthesiologica Scandinavica, Vol. 32, No. 8, 1988, pp. 607-613. http://dx.doi.org/10.1111/j.1399-6576.1988.tb02796.x

[20] P. S. Pagel and D. C. Warltier, "Anesthetics and Left Ventricular Function,” In: D. C. Waltier, Ed., Ventricular Function, Williams \& Wilkins, Baltimore, 1995, pp. 213252. 